\title{
Suppression of ongoing experimental myasthenia by oral treatment with an acetylcholine receptor recombinant fragment
}

\author{
Sin-Hyeog Im, ${ }^{1}$ Dora Barchan, ${ }^{1}$ Sara Fuchs,,${ }^{1}$ and Miriam C. Souroujon ${ }^{1,2}$ \\ ${ }^{1}$ Department of Immunology, The Weizmann Institute of Science, Rehovot 76100, Israel \\ ${ }^{2}$ The Open University, Tel-Aviv 61392, Israel
}

Address correspondence to: Sara Fuchs, Department of Immunology, The Weizmann Institute of Science, Rehovot 76100, Israel. Phone: 972-8-9342618; Fax: 972-8-9344141; E-mail: lifuchs@wiccmail.weizmann.ac.il.

Received for publication August 10, 1999, and accepted in revised form November 2, 1999.

\begin{abstract}
Myasthenia gravis (MG) is an autoimmune disorder in which the nicotinic acetylcholine receptor (AChR) is the major autoantigen. In an attempt to develop an antigen-specific therapy for MG, we administered a nonmyasthenogenic recombinant fragment of AChR orally to rats. This fragment,

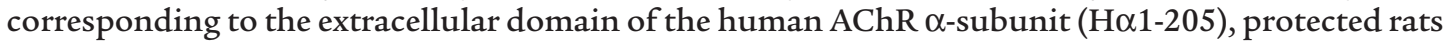
from subsequently induced experimental autoimmune myasthenia gravis (EAMG) and suppressed ongoing EAMG when treatment was initiated during either the acute or chronic phases of disease. Prevention and suppression of EAMG were accompanied by a significant decrease in AChR-specific humoral and cellular responses. The underlying mechanism for the $\mathrm{H} \alpha 1-205$-induced oral tolerance seems to be active suppression, mediated by a shift from a T-helper 1 (Th1) to a Th2/Th3 response. This shift was assessed by changes in the cytokine profile, a deviation of anti-AChR IgG isotypes from IgG2 to IgG1, and a suppressed AChR-specific delayed-type hypersensitivity response. Our results in experimental myasthenia suggest that oral administration of AChR-specific recombinant fragments may be considered for antigen-specific immunotherapy of myasthenia gravis.
\end{abstract}

J. Clin. Invest. 104:1723-1730 (1999).

\section{Introduction}

Myasthenia gravis (MG) and experimental autoimmune myasthenia gravis (EAMG) are autoimmune disorders in which the nicotinic acetylcholine receptor (AChR) at the neuromuscular junction is the major autoantigen. EAMG mimics human MG in its clinical and immunopathologic manifestations and is a reliable model for the investigation of therapeutic strategies for myasthenia $(1,2)$. MG and EAMG are antibody-mediated autoimmune disorders, but $\mathrm{T}$ cells have been demonstrated to have a pivotal role in the autoimmune response (3-8).

Current treatment of MG involves general immunosuppression, which may have adverse side effects, whereas the ideal therapy should specifically eliminate the autoimmune response to AChR without affecting the function of the entire immune system (9). Our first successful attempt for antigen-specific immunotherapy of EAMG was performed by denatured AChR (10). In that study, we showed that intradermal injection of a reduced and carboxy methylated derivative of Torpedo AChR (RCM$\mathrm{AChR}$ ) in CFA could prevent the induction of EAMG in rabbits and immunosuppress an ongoing disease. Other antigen-specific approaches for suppression of EAMG, based on AChR-derivatives or aimed at the pathogenic autoantibodies, have been reported by us and others (11-17).

Exposure of mucosal surfaces to antigen is known to lead to specific systemic tolerance (18). This observation has encouraged attempts to induce mucosal tolerance to autoantigens in order to modulate a variety of experimental autoimmune diseases (19-26) including EAMG (27-30).

Previous studies focusing on prevention of EAMG have shown that oral or nasal administration of Torpedo AChR or its $\mathrm{CD}^{+}{ }^{+} \mathrm{T}$-cell epitopes before induction of EAMG prevented the clinical manifestation of the disease and suppressed cellular and humoral responses to AChR (27-30). However, to our knowledge, there is no report of a successful treatment for ongoing EAMG, especially in a chronic disease model. Moreover, Torpedo AChR, being a highly immunogenic and xenogeneic protein available in limited amounts, is not likely to be considered for therapeutic purposes in humans (2).

We have chosen to use a nonmyasthenogenic, readily available recombinant fragment of mammalian AChR, administered orally in order to suppress ongoing EAMG in rats. Administration of fragment Ho1205 , corresponding to the extracellular domain of the human AChR $\alpha$-subunit, not only prevented the subsequent induction of EAMG but also immunosuppressed an already existing disease in rats, even when treatment was initiated at the chronic phase of disease. The underlying mechanism of this tolerance seems to be a shift from a Th1- to a Th2/Th3-regulated AChR-specific immune response. 


\section{Methods}

Antigen preparation. AChR was purified from the electroplax tissue of Torpedo californica by affinity chromatography on Naja naja siamensis $\alpha$-cobrotoxin, as described previously (31). The recombinant fragment H $\alpha 1-205$, was synthesized by PCR on cDNA prepared from total RNA of the human TE671 cell line and cloned in the pET8C expression vector. The protein, present in inclusion bodies, was solubilized by $9 \mathrm{M}$ urea followed by serial dialyses in $50 \mathrm{mM}$ Tris buffer ( $\mathrm{pH}$ 8.0).

Induction of EAMG and clinical evaluation. Female Lewis rats, 6 to 7 weeks of age, were immunized once in both hind footpads by subcutaneous injection of Torpedo AChR $(45 \mu \mathrm{g})$ emulsified in CFA containing additional Mycobacterium tuberculosis (1 mg/rat; Difco Laboratories, Detroit, Michigan, USA). Clinical severity of EAMG was scored on a scale of 0-4 according to Lennon et al. (32). Animals were evaluated weekly for 7-10 weeks after immunization with Torpedo AChR in prevention or acute phase-treatment experiments and for up to 15 weeks in chronic phase-treatment experiments.

Induction of oral tolerance. Feeding with $\mathrm{H} \alpha 1-205$ or ovalbumin (OVA; as control) was initiated either before immunization with Torpedo AChR or at the acute or chronic phases of EAMG. In prevention experiments, rats were fed 6 times with 2-day intervals. For oral treatment at the acute phase of EAMG, feeding was initiated 8 days after disease induction, and for treatment at the chronic phase, feeding was initiated 7 weeks after disease induction and continued twice a week. The amount of $\mathrm{H} \alpha 1-205$ and OVA was $600 \mu \mathrm{g} /$ dose per rat in $1 \mathrm{~mL}$ Tris buffer (50 mM [pH 8.0]) in all treatment protocols.

Antibody assays. Serum anti-Torpedo AChR and anti-H $\alpha 1-205$ antibody levels were assayed by ELISA as described previously (33). Antibodies to rat muscle AChR were measured by radioimmunoassay with a crude rat muscle extract in which the AChR was specifically labeled by ${ }^{125}$ I- $\alpha$-bungarotoxin ( $\alpha$-BTX) (34). Results are expressed as nanomoles of antibody per liter of serum.

Determination of AChR-specific IgG isotypes was performed as described previously (35). Microtiter plates were coated with Ho1-205 $(100 \mu \mathrm{L} ; 20 \mu \mathrm{g} / \mathrm{mL})$ and reacted with $100 \mu \mathrm{L}$ of the tested serum samples at proper dilutions (1:250 for IgG1 and IgG2a; 1:10 for IgG2b and IgG2c). Biotinylated mouse mAb's to rat IgG isotypes $(100 \mu \mathrm{L}$ at 1:1,000; Caltag Laboratories Inc., Burlingame, California, USA) were added for 2 hours at room temperature, and bound antibodies were detected by activity of alkaline phosphatase-conjugated Extravidin (Sigma Israel Chemicals, Rehovoto, Israel).

Delayed-type bypersensitivity. Assessment of delayed-type hypersensitivity (DTH) was performed by subcutaneous injection into the contralateral ear with Torpedo AChR $(40 \mu \mathrm{g})$, purified protein derivative of $M$. tuberculosis (PPD; 1:2,000; Statens Serum Institute, Copenhagen, Denmark) or PBS. The difference in ear lobe thickness before challenge and 48 hours after challenge was recorded for each animal. Results are expressed as the mean of 5 animals for each experimental group \pm SEM.
Lymphocyte assays. Proliferation of lymph node cells (LNCs) from treated rats was performed essentially as described elsewhere $(35,36)$. Draining lymph nodes were removed 6-8 weeks after immunization in prevention and acute phase-treatment experiments and 10-13 weeks after immunization in chronic phase-treatment experiments. Proliferation was assessed by measuring $\left[{ }^{3} \mathrm{H}\right]$ thymidine $(0.5 \mu \mathrm{Ci} /$ well $)$ incorporation during the last 18 hours of a 4-day culture period. Results are expressed as $\Delta \mathrm{cpm}$ (obtained by subtraction of background of unstimulated cultures from stimulated LNCs).

The presence of IL-2 in supernatants from LNC cultures was assayed by its ability to induce proliferation of the CTLL, IL-2-dependent (non-IL-4-responsive) cell line, as described previously $(35,37)$. Essentially, supernatants $(50 \mu \mathrm{L})$ of LNCs incubated in the presence of Torpedo $\operatorname{AChR}(0.25 \mu \mathrm{g} / \mathrm{mL})$ or concanavalin $\mathrm{A}(2 \mu \mathrm{g} / \mathrm{mL})$ for 24 hours were added to CTLL cells. $\left[{ }^{3} \mathrm{H}\right]$ Thymidine pulse was performed during the last 20 hours of a 2-day culture period. Results are expressed as $\Delta \mathrm{cpm}$.

T-cell anergy was tested as described previously (36). Essentially, single-cell suspensions of draining LNCs were incubated for 5 days with or without recombinant rat IL2 (rIL-2; 2-10 ng/mL; R\&D Systems Inc., Minneapolis, Minnesota, USA). The cells were then transferred to 96well plates $\left(5 \times 10^{5}\right.$ cells per well $)$ and incubated with Torpedo AChR. Proliferation was assessed by measuring $\left[{ }^{3} \mathrm{H}\right]$ thymidine uptake as already described here.

Adoptive transfer of splenocytes. Adoptive transfer experiments were performed as described previously (38). Donor rats were fed with $\mathrm{H} \alpha 1-205$ or OVA $(0.6 \mathrm{mg} / \mathrm{dose})$, 6 times at 2- or 3-day intervals. Their spleens were removed 3 days after the last feeding, and splenocytes $\left(10^{8}\right)$ were injected intraperitoneally to naive syngeneic recipient rats irradiated with $2.6 \mathrm{~Gy} / 2$ minutes. Recipients were immunized with Torpedo $\mathrm{AChR}(45 \mu \mathrm{g})$ in CFA 2 hours after cell transfer and were evaluated for clinical symptoms of EAMG up to 8 weeks after immunization.

Cytokine level determination. PCR-ELISA was used to assess the levels of mRNA specific for IL-2, IL-10, IL-12, IFN- $\gamma$, and TGF- $\beta$. RNA extraction, cDNA synthesis, and RT-PCR in the presence of digoxigenin-dNTP (DIGdNTP) were performed as described by Zipris et al. (39) with some modification suggested by the manufacturer of the PCR-ELISA kit (Boehringer-Mannheim). The sequences of primer pairs specific for the IL-2, IL-10, and IFN- $\gamma$ were the same as previously reported (39). The sequences for other primers used are as follows: IL-2 internal primer (5'-CTTGCCCA AGCAGGCCACAGAATT-3'); IL-10 internal primer (5'-GCCCCAGAACCATGGCCCA-3'); IL-12p40 sense primer (5'-TGGTTTGCCATGGTTTTGCTGG-3'), IL-12p40 antisense primer (5'AGGAATGGGGAGTGCTCCAGGA-3') and IL-12p40 internal primer (5'-TCCCC TGAGTCTCGGGCGGTGACA-3'). IFN- $\gamma$ internal primer $\left(5^{\prime}\right.$-CATGGATGCTATGGAAG GAAAGAGC-3'); TGF- $\beta$ sense primer (5'-ACAACAGCACCCGCCACCGGGTGGC-3'), TGF- $\beta$ antisense primer $\left(5^{\prime}\right.$-GCCCTTGGGCTCGTGGATCCACTTCC-3') and TGF- $\beta$ internal 
primer (5'-GTCACCCGCGTGCTAATGGTGGACC-3'). The internal primers were all Biotinylated. The amplified DIG-labeled PCR products were quantified using a PCRELISA kit (Roche Molecular Biochemicals, Mannheim, Germany). They were then denatured and hybridized to the suitable cytokine-specific biotinylated internal primers for 3 hours at $37^{\circ} \mathrm{C}$ with constant shaking. The DIG-labeled PCR product/biotinylated probe hybrids were immobilized on streptavidin-coated 96-well ELISA plates. After washing, the bound PCR products were detected with a peroxidase-conjugated anti-DIG antibody. PCR products were viewed with the peroxidase substrate ABTS, and signals were quantified by absorbance at $405 \mathrm{~nm}$ (40).

\section{Results}

Effect of oral pretreatment with Ho1-205 on EAMG. The main goal of this study was to test the ability of an orally administered recombinant fragment corresponding to the extracellular domain of a mammalian AChR $\alpha$ subunit, to modulate ongoing EAMG. However, we first tested the effectiveness of this fragment to prevent EAMG subsequently induced in rats by Torpedo AChR. The fragment used in the study, $\mathrm{H} \alpha 1-205$, corresponds to residues 1-205 of the human AChR $\alpha$-subunit, which is $96 \%$ homologous to the corresponding rat AChR fragment. When injected with CFA into the footpads of rats, Ho1-205 does not induce any signs of myasthenia (S.$\mathrm{H}$. Im et al., unpublished study). Rats were fed with Ho1-205 (0.6 mg/dose) or OVA as control 3 times a week for 2 weeks. Two days after the last feeding, rats were immunized with Torpedo AChR for induction of EAMG and followed up clinically for 8 weeks. As shown in Table 1 and Figure 1, significant protection against EAMG was observed in rats pretreated with $\mathrm{H} \alpha 1-205$. At all times tested, the mean clinical score of EAMG in groups pretreated with Ho1-205 was lower than that of the OVA-pretreated group (Figure 1). Eight weeks after induction of EAMG, all 10 rats in the control group were sick (mean clinical score: 2.8 ), whereas among rats pretreated with Ho1-205, 70\% were completely protected against EAMG and did not have symptoms of EAMG. The remaining 30\% showed mild symptoms compared with the control group. The mean clinical score in the Ho1-205-treated rats was 0.5.



Figure 1

Pretreatment by $\mathrm{H} \alpha 1-205$ protects rats against EAMG. Rats were pretreated 6 times with H $\alpha 1-205$ or with OVA as control, over a period of 2 weeks and subsequently immunized with Torpedo AChR. Mean EAMG clinical scores were followed up until 8 weeks after injection of Torpedo AChR. Representative of 6 independent experiments. ${ }^{*} P<0.005$.

Quantitative parameters of disease included assessment of body weight and muscle AChR content. Rats in the control OVA-pretreated group lost $8.8 \pm 9.2 \mathrm{~g} / \mathrm{rat}$ at 3-8 weeks after induction of EAMG, whereas rats in the Ho1-205-pretreated group gained at the same time 9.8 $\pm 5.3 \mathrm{~g} / \mathrm{rat}$ (Table 1 ). The analysis of AChR content verified the observation of clinical symptoms. Rats in the control OVA-pretreated group lost $60 \%$ of their muscle AChR content, whereas rats in the Ho1-205-pretreated group lost only $8.5 \%$ of their muscle AChR.

Oral administration of H $\alpha 1-205$ had also an effect on AChR-specific humoral and cellular immune responses. There was a significant decrease in the level of antibodies to rat muscle AChR in H 1 1-205-pretreated rats when measured 8 weeks after immunization with Torpedo AChR (Table 1). Antibody levels against the recombinant fragment itself were similarly decreased (data not shown). There was also a marked reduction in the AChR-specific proliferative T-cell response and IL-2 production (Table 1). The DTH response to Torpedo AChR, tested 1 week after disease induction, was specifically reduced in $\mathrm{H} \alpha 1-205$-treated rats compared with OVA-treated rats $\left(30 \pm 5 \mathrm{~mm} \times 10^{-2}\right.$ and $97 \pm 9 \mathrm{~mm}$ $\times 10^{-2}$, respectively). There was no difference between the groups in the DTH response to PPD and PBS. These data indicate that oral pretreatment with $\mathrm{H} \alpha 1$ -

Table 1

Prevention of EAMG by oral pretreatment with AChR fragment

\begin{tabular}{|c|c|c|c|c|c|c|c|c|c|c|c|}
\hline \multirow[t]{2}{*}{ Treatment } & \multicolumn{5}{|c|}{ Clinical score (no./total) ${ }^{\mathrm{A}}$} & $\Delta$ weight & \multicolumn{2}{|c|}{ AChR content ${ }^{B}$} & \multirow{2}{*}{$\begin{array}{l}\text { Anti-rat } \\
\text { AChR } \\
(\mathrm{nM})^{\mathrm{C}}\end{array}$} & \multirow{2}{*}{$\begin{array}{c}\text { T-cell } \\
\text { proliferation } \\
(\mathrm{cpm})^{\mathrm{D}}\end{array}$} & \multirow{2}{*}{$\begin{array}{l}\text { IL-2- } \\
\text { dependent } \\
\text { proliferation } \\
(\mathrm{cpm})^{\mathrm{E}}\end{array}$} \\
\hline & 0 & 1 & 2 & 3 & 4 & $\begin{array}{c}\text { 3-8 weeks } \\
(\mathrm{g})\end{array}$ & $\begin{array}{l}\text { (fmol/mg } \\
\text { protein) }\end{array}$ & (\%) & & & \\
\hline Control (OVA) & $0 / 10$ & $1 / 10$ & $3 / 10$ & $3 / 10$ & $3 / 10$ & $-8.8 \pm 9.2$ & $16.5 \pm 5.1$ & 39.8 & $70 \pm 8$ & $7,200 \pm 1200$ & $11,000 \pm 800$ \\
\hline H $\alpha 1-205$ & $7 / 10$ & $1 / 10$ & $2 / 10$ & $0 / 10$ & $0 / 10$ & $+9.8 \pm 5.3$ & $38.0 \pm 5.5$ & 91.5 & $25 \pm 3$ & $810 \pm 210$ & $1,800 \pm 400$ \\
\hline
\end{tabular}

${ }^{A}$ Evaluated 8 weeks after the induction of EAMG. BMuscle AChR content (femtomoles per milligram of protein) in normal age-matched rats was $41.5 \pm$ $2.7 \mathrm{fmol} / \mathrm{mg}$ protein and was referred to as the $100 \%$ value for this experiment. CPresented as nanomoles of $\alpha$-BTX-binding sites precipitated by $1 \mathrm{~L}$ serum. DIn response to Torpedo AChR $(0.25 \mu \mathrm{g} / \mathrm{mL})$. EProliferation of the IL-2-dependent CTLL cell line in response to supernatants of LNCs stimulated by Torpedo AChR $(0.25 \mu \mathrm{g} / \mathrm{mL})$. 



Figure 2

Treatment of acute and chronic EAMG. Torpedo AChR was injected to induce EAMG, and rats were treated twice a week by oral administration of $\mathrm{H} \alpha 1-205$ or OVA starting at the acute phase, 8 days after disease induction (a), or at the chronic phase, 7 weeks after disease induction (b). Treatments were performed as described in Methods. Representative of 3 independent experiments. ${ }^{*} P<0.001$.

205 induced a suppressed T-cell response to AChR both in vitro and in vivo.

Effect of oral treatment with H 1 1-205 on ongoing EAMG. To investigate whether oral administration of the AChR-derived recombinant fragment $\mathrm{H} \alpha 1-205$ is also potentially suitable for treatment of myasthenia, we tested its effect on an already existing disease. Oral treatment was first performed at the acute phase of EAMG, which usually starts 7-10 days after the immunization of rats with Torpedo AChR. For that, admin- istration of H $\alpha 1-205$ ( $0.6 \mathrm{mg} /$ dose $)$ was initiated 8 days after induction of EAMG and was continued twice a week for 8 weeks. Given this treatment, all 10 rats in the control OVA-treated group got sick and 6 of 10 died of EAMG in the first 10 weeks after disease induction. On the other hand, in the Ho1-205-treated group, 6 of 10 rats were completely healthy or mildly sick and none of the rats died (Table 2). When assessed 10 weeks after AChR injection, the mean clinical score of Ho1205 -treated rats was 1.2 compared with 3.3 in the OVAtreated rats (Figure 2a).

Clinical signs characteristic of the chronic phase of EAMG usually appear about 4 weeks after immunization with Torpedo AChR. Rats presenting mild EAMG (clinical score 1), at 7 weeks after EAMG induction, were selected and treated twice a week for 3 more weeks. This treatment protocol had an effect on the severity of the disease (Figure $2 \mathrm{~b}$ and Table 2). In the OVA-treated group, there was an aggravation in the severity of EAMG: 7 weeks after the initiation of treatment, 2 of 6 rats died and 2 others were severely sick (mean clinical score: 2.3). However, in the Ho1205-treated group, none of the rats died and 3 of 6 rats recovered completely (Table 2 ). The suppressive effect of feeding with $\mathrm{H} \alpha 1-205$ on EAMG lasted at least up to 4 weeks after it has been discontinued, when rats were sacrificed for further analysis.

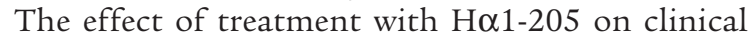
symptoms at the acute and chronic phases of EAMG was also corroborated by the analyses of AChR content and body weight changes (Table 2). Rats in the control OVA-treated group (all presenting EAMG) lost 59\% and $65 \%$ of their muscle AChR content in the acute phaseand chronic phase-treatment protocols, respectively. In contrast, rats treated by $\mathrm{H} \alpha 1-205$ during the acute and chronic phases of EAMG lost only $10 \%$ and $17 \%$ of their muscle AChR, respectively (Table 2).

The effect of oral administration of Ho1-205 on EAMG was also accompanied by suppression in AChRspecific humoral and cellular immune responses. Rats

Table 2

Treatment of ongoing EAMG by oral treatment with AChR fragment

\begin{tabular}{|c|c|c|c|c|c|c|c|c|c|c|c|}
\hline \multirow[t]{2}{*}{ Treatment } & \multicolumn{5}{|c|}{ Clinical score $(\text { no./total })^{\mathrm{A}}$} & $\Delta$ weight $^{\mathrm{B}}$ & \multicolumn{2}{|c|}{ AChR content ${ }^{C}$} & \multirow{2}{*}{$\begin{array}{l}\text { Anti-rat } \\
\text { AChR } \\
(\mathrm{nM})^{\mathrm{D}}\end{array}$} & \multirow{2}{*}{$\begin{array}{c}\text { T-cell } \\
\text { proliferation } \\
(\mathrm{cpm})^{\mathrm{E}}\end{array}$} & \multirow{2}{*}{$\begin{array}{l}\text { IL-2- } \\
\text { dependent } \\
\text { proliferation } \\
(\mathrm{cpm})^{\mathrm{F}}\end{array}$} \\
\hline & 0 & 1 & 2 & 3 & 4 & (g) & $\begin{array}{l}(\mathrm{fmol} / \mathrm{mg} \\
\text { protein) }\end{array}$ & $(\%)$ & & & \\
\hline \multicolumn{12}{|c|}{ Acute phase } \\
\hline $\begin{array}{l}\text { OVA } \\
\text { H } \alpha 1-205\end{array}$ & $\begin{array}{l}0 / 10 \\
4 / 10\end{array}$ & $\begin{array}{l}0 / 10 \\
2 / 10\end{array}$ & $\begin{array}{l}3 / 10 \\
2 / 10\end{array}$ & $\begin{array}{l}1 / 10 \\
2 / 10\end{array}$ & $\begin{array}{l}6 / 10 \\
0 / 10\end{array}$ & $\begin{array}{c}-21.9 \pm 19.5 \\
+9.6 \pm 19.3\end{array}$ & $\begin{array}{l}18.6 \pm 1.9 \\
41.0 \pm 2.0\end{array}$ & $\begin{array}{l}41 \\
90\end{array}$ & $\begin{array}{c}116 \pm 12 \\
30 \pm 5\end{array}$ & $\begin{array}{c}7,250 \pm 1,100 \\
1,050 \pm 450\end{array}$ & $\begin{array}{c}7,800 \pm 1,800 \\
1,100 \pm 670\end{array}$ \\
\hline \multicolumn{12}{|c|}{ Chronic phase } \\
\hline $\begin{array}{l}\text { OVA } \\
\text { H } \alpha 1-205\end{array}$ & $\begin{array}{l}1 / 6 \\
3 / 6\end{array}$ & $\begin{array}{l}1 / 6 \\
1 / 6\end{array}$ & $\begin{array}{l}1 / 6 \\
1 / 6\end{array}$ & $\begin{array}{l}1 / 6 \\
1 / 6\end{array}$ & $\begin{array}{l}2 / 6 \\
0 / 6\end{array}$ & $\begin{array}{c}-8.5 \pm 31.8 \\
+18.2 \pm 10.8\end{array}$ & $\begin{array}{l}16.2 \pm 2.0 \\
37.6 \pm 3.8\end{array}$ & $\begin{array}{l}35 \\
83\end{array}$ & $\begin{array}{l}94 \pm 8 \\
16 \pm 3\end{array}$ & $\begin{array}{c}8,800 \pm 1,980 \\
1,500 \pm 450\end{array}$ & $\begin{array}{c}6,800 \pm 1,200 \\
850 \pm 350\end{array}$ \\
\hline
\end{tabular}

${ }^{A}$ Evaluated at 10 or 14 weeks after the induction of EAMG in the acute phase- or chronic phase-treatment protocols, respectively. ${ }^{\mathrm{B}}$ Evaluated between $4-10$ weeks or between 8-14 weeks at the acute phase- or chronic phase-treatment protocols, respectively. ${ }^{\mathrm{C}}$ Muscle AChR content (femtomoles per milligram of protein) in normal, age-matched rats was $45.5 \pm 3.0 \mathrm{fmol} / \mathrm{mg}$ protein and was referred to as the $100 \%$ value for this experiment. ${ }^{\mathrm{D}}$ Derived from nanomoles of

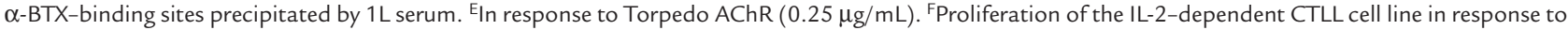
supernatants of LNCs stimulated by Torpedo AChR $(0.25 \mu \mathrm{g} / \mathrm{mL})$. 


\section{Figure 3}

Exogenous IL-2 does not restore the suppressed T-cell response. AChR-specific lymphocyte proliferation of rats treated by H $1-205$ or OVA at the acute phase of EAMG was measured before (a) and after a 5-day culture in the absence (b) or presence (c) of rat rIL-2. Results are expressed as mean stimulation index (counts per minute incorporated by stimulated cells per counts per minute incorporated by unstimulated cells). Biologic activity of rIL-2 was verified by the proliferative response of the CTLL cell line. Representative of 3 independent experiments. ${ }^{*} P<0.005$. treated orally with $\mathrm{H} \alpha 1-205$ had a marked decrease in their anti-self AChR antibody levels when treated at the acute $(73 \%$ reduction) or chronic ( $80 \%$ reduction) phase of EAMG (Table 2). The antibody titers to self AChR seem to correlate with the clinical symptoms better than the antibody titers toward the xenogeneic Torpedo $\mathrm{AChR}$, in which there were no significant differences between the groups. There was also a marked decrease in the AChR-specific proliferative T-cell response and IL-2 production after treatment initiated either at the acute or chronic phases of EAMG (Table 2).

Possible mechanisms of oral tolerance. Our next goal was to analyze the possible mechanisms involved in suppression of EAMG by oral administration of the recombinant AChR fragment. To find out whether clonal anergy is induced by oral tolerization, we tested the ability of exogenous IL-2 to reverse the suppression of AChR-specific T-cell proliferative responses observed in rats treated by $\mathrm{H} \alpha 1-205$ during the acute phase of EAMG (Figure $2 \mathrm{a}$ and Table 2). LNCs from Ho1-205-treated rats were preincubated in the presence of rIL-2 for 5 days, and proliferation was assessed. As seen in Figure 3, preincubation with exogenous rIL-2 did not restore the suppressed

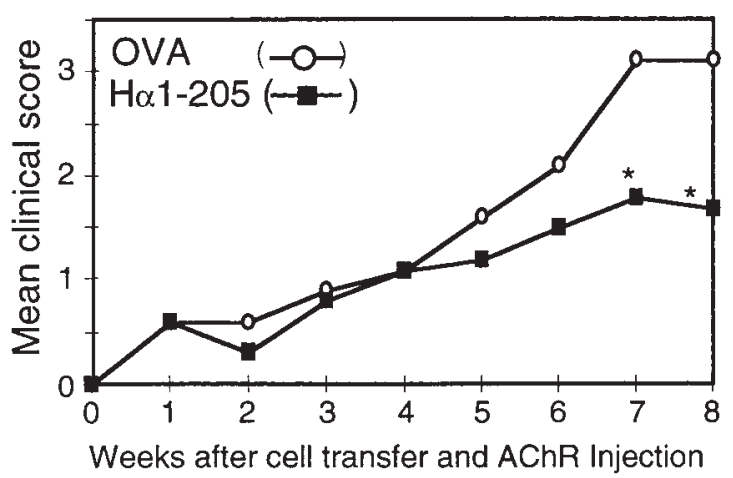

Figure 4

Protection can be adoptively transferred by splenocytes from $\mathrm{H} \alpha 1$ 205-treated rats. Splenocytes from Ho1-205- or OVA-treated rats were injected intraperitoneally to irradiated naive recipients that were subsequently immunized with Torpedo AChR to induce EAMG. Clinical symptoms in recipient rats were followed up for 8 weeks after immunization. Each group consisted of 10 rats. Representative of 3 independent experiments. ${ }^{*} P<0.002$.

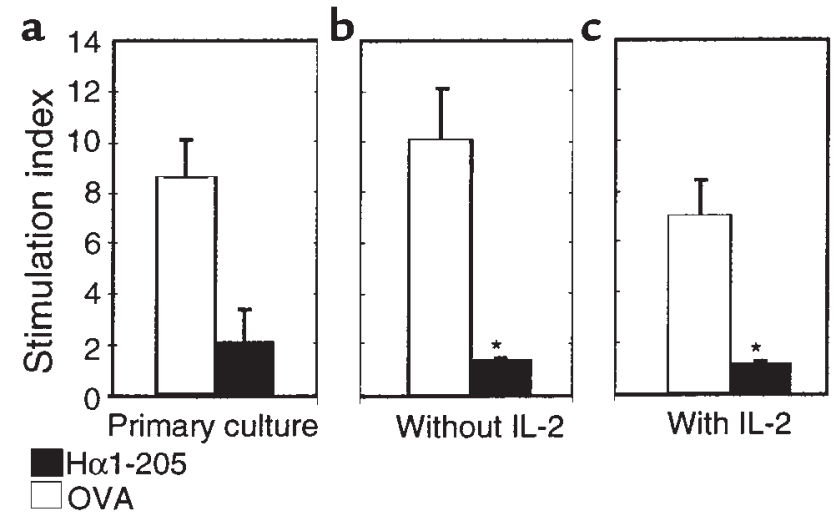

AChR-specific proliferative response. These observations suggest that clonal anergy is probably not involved in the tolerance induced by oral feeding with the AChR-derived recombinant fragment.

To investigate whether oral administration of the AChR-derived recombinant fragment results in generation of active suppression, we attempted to adoptively transfer the protection against EAMG from $\mathrm{H} \alpha 1$ 205-treated rats to naive recipient rats. Splenocytes were harvested from rats that were fed 6 times over a period of 2 weeks with either H $\alpha 1-205$ or OVA and injected intraperitoneally $\left(10^{8}\right.$ per rat $)$ into naive syngeneic recipient rats irradiated with $2.6 \mathrm{~Gy} / 2$ minutes. Recipient rats were immunized 2 hours later with Torpedo AChR for induction of EAMG. A protective effect of the splenocytes transferred from $\mathrm{H} \alpha 1-205$-treated rats was observed in the recipient rats and was especially evident at their chronic phase of disease (Figure 4). At 8 weeks after transfer, recipients of splenocytes from Ho1-205-treated rats had a mean clinical score of 1.6, compared with 3.1 in rats that received splenocytes from control OVA-treated donors. The protective effect provided by the adoptive transfer of splenocytes from

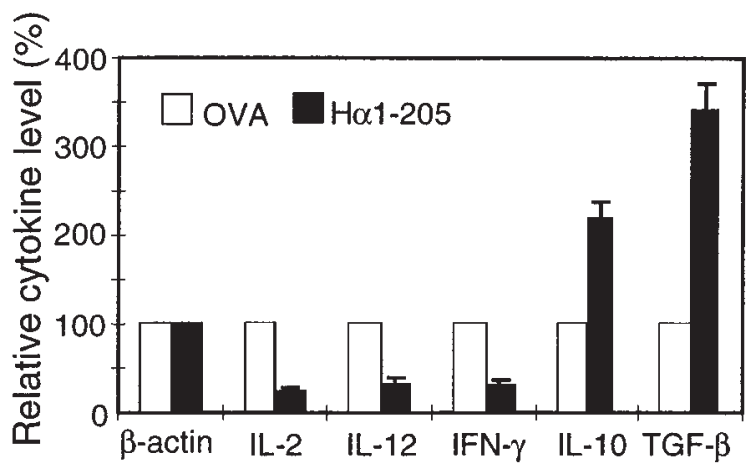

Figure 5

Shift in the cytokine profile from a Th1 to a Th2/Th3 type. LNCs from rats treated at the acute phase of EAMG with H 1 1-205 or OVA were cultured for 2 days in the presence of AChR, and mRNA was prepared. The mRNA expression level of cytokines (and of $\beta$-actin as control) was determined by PCR-ELISA, and the data are expressed as the relative value of the Ho1-205-treated group compared with the OVA-treated group. 

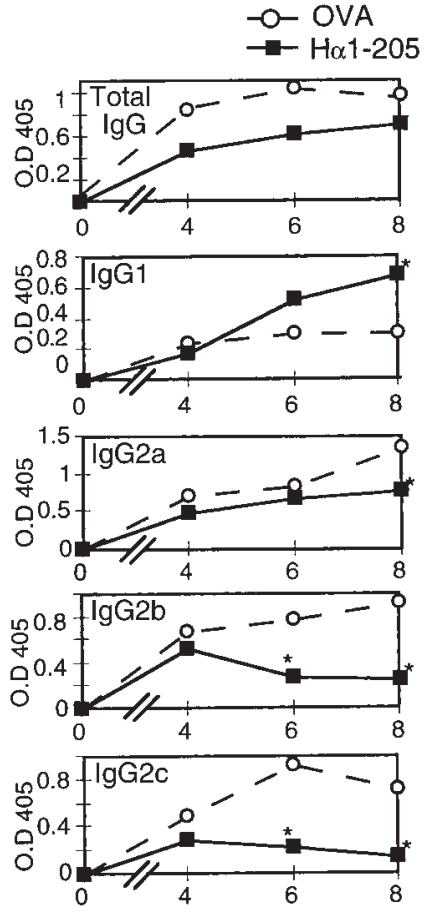

\section{Figure 6}

Isotype profile of AChR-specific IgGs. Rats immunized with Torpedo AChR and treated at the acute phase of EAMG with Ho1-205 or OVA were bled every 2 weeks for up to 8 weeks after immunization. The anti-H $1-205$ IgG isotype levels were determined by ELISA. ${ }^{*} \mathrm{P}<0.002$.

H $\alpha 1-205$-treated rats suggests an involvement of active suppression in the tolerance induced by oral administration of $\mathrm{H} \alpha 1-205$.

Because active suppression is mediated by regulatory $\mathrm{T}$ cells that secrete distinct cytokines, we examined the cytokine mRNA expression level in treated rats. Draining LNCs were removed 5-7 weeks after EAMG induction from rats treated with OVA or $\mathrm{H} \alpha 1-205$ at the acute phase of disease and were cultured for 48 hours in the presence of Torpedo AChR. Total RNA was then prepared from the cells and subjected to PCR-ELISA with cytokine-specific primers. As shown in Figure 5, oral treatment with $\mathrm{H} \alpha 1-205$ resulted in suppression of Th1type (IL-2, IL-12, and IFN- $\gamma$ ) cytokine mRNA levels and in upregulation of Th2-type (IL-10) or Th3-type (TGF$\beta)$ cytokine mRNA levels. These results suggest that oral treatment with $\mathrm{H} \alpha 1-205$ induced a shift from a Th1toward a Th2/Th3-regulated anti-AChR response.

The observed shift in the T-cell subpopulations of rats treated by $\mathrm{H} \alpha 1-205$ was also reflected in an altered IgG isotype profile of anti-AChR autoantibodies. The IgG isotype levels of antibodies to $\mathrm{H} \alpha 1-205$ were measured every 2 weeks after induction of EAMG. The homology between this human AChR-derived fragment and its homologue in the rat is $96 \%$. Therefore, antibodies directed to fragment $\mathrm{H} \alpha 1-205$ could represent the antibody response toward self rat muscle AChR. As seen in Figure 6, total anti-H $\alpha 1-205$ IgG levels were lower in Ho1-205-treated rats than in the con- trol OVA-treated rats. On the other hand, the levels of IgG1, but not of IgG2, in $\mathrm{H} \alpha 1-205$-treated rats were higher than in control rats. All IgG2 subtypes (in particular IgG2b and IgG2c) were lower in the Ho1205-treated rats compared with controls (Figure 6). This may suggest that IgG2b and IgG2c are involved in the immunopathogenic process taking place in rat EAMG. IgG2b has been previously suggested to be involved in rat EAMG (41), but to our knowledge, there is no former information concerning the role of $\operatorname{IgG} 2 \mathrm{c}$ in rat EAMG. Similar shifts in the AChR-specific IgG isotype profile were observed also when oral treatment was given before EAMG induction or when oral treatment was initiated during the chronic phase of EAMG (data not shown). In the rat, IgG1 production is thought to be regulated by a Th2-type response, whereas IgG2 isotypes are regulated by a Th1 response. The observed shift in the anti-AChR isotype profile in $\mathrm{H} \alpha 1$ 205-treated rats may thus reflect a deviation from a Th1- to a Th2-regulated response. Taken together, the shift in cytokine profile, the deviation in the anti-AChR IgG isotypes, and the reduced DTH response (a known Th1-regulated response), in Ho1-205-treated rats, suggest that the oral tolerance induced by $\mathrm{H} \alpha 1-205$ involves a shift from a Th1- to a Th2/Th3-regulated immune response to the autoantigen.

\section{Discussion}

In this study, we have demonstrated that oral administration of a recombinant fragment corresponding to the extracellular domain of the human AChR $\alpha$-subunit is capable of protecting rats against subsequently induced EAMG and suppressing an already ongoing acute as well as chronic disease. Our results suggest that the mechanism of this oral tolerance involves active suppression and a shift in the AChR-specific immune response from Th1 to Th2/Th3 regulation, as reflected by changes in the cytokine profile; in a decreased DTH response to AChR; and in a switch in anti-AChR specific IgG isotypes from IgG2 to IgG1.

Mucosal exposure was chosen as the route of administration, as it is known to result in systemic tolerance to the specific tolerogen. We have recently reported on modulation of EAMG by nasal administration of recombinant fragments corresponding to the extracellular domain of the human AChR $\alpha$-subunit expressed as fusion proteins, either before disease induction or at the acute phase of disease (35). Encouraged by the data presented in our previous study, we have set out to attempt treatment of EAMG by the oral route, also during its chronic phase, which parallels the situation of patients with MG at the time of their diagnosis. In the present study, we used a recombinant AChR fragment without any fusion partner, rendering it more suitable for application in humans.

Oral tolerance has been implied in other autoimmune diseases to act via inhibition of Th1 responses either by deletion or anergy of the undesirable autoreactive cells or by their active suppression via Th2/Th3- 
secreted regulatory cytokines. These mechanisms are not mutually exclusive and may occur simultaneously. In oral tolerance, the dose of antigen administered is the primary factor in determining which mechanism predominates. Low antigen doses are known to favor active suppression-that is, the induction of antigenspecific cells that suppress the activity of other immune cells by secretion of anti-inflammatory cytokines such as TGF- $\beta$, IL-4, and IL-10. On the other hand, high antigen doses drive specific $T$ cells into a state of clonal anergy or clonal deletion (18). In this study, we treated rats by $5-20$ oral feedings of $0.6 \mathrm{mg} /$ dose (depending on the treatment protocol) and have demonstrated that active suppression is involved in tolerance induction. This was shown by the ability of splenocytes, isolated from rats treated orally with H $\alpha 1-205$, to adoptively transfer protection against EAMG to naive rats. We did not observe clonal anergy in Ho1-205-treated rats, as IL-2 failed to restore their suppressed T-cell proliferative responsiveness to AChR. We are currently studying the possible involvement of clonal deletion. Most of the previous studies in EAMG have focused on attempts to prevent EAMG. These studies have shown that mucosal administration of Torpedo AChR before immunization with AChR prevents the clinical manifestation of the disease and suppresses cellular and humoral responses to the AChR (27-29). When feeding with Torpedo AChR was performed during the acute phase of EAMG, it led to the elicitation of antibodies to Torpedo and to an increase in autoantibody titers to self muscle AChR $(41,42)$. The priming effect on autoantibody levels induced by feeding with the xenogeneic and highly immunogenic Torpedo AChR and its limited availability hamper its application for therapeutic purposes. This suggests that a syngeneic, modified, less immunogenic, and easily available tolerogen is required for immunotherapy of myasthenia in humans. The recombinant fragment of the human AChR $\alpha$-subunit used in this study, meets these requirements: it is of mammalian origin, available in large amounts, and seems to be safe since it does not induce any humoral anti-AChR response or signs of EAMG even when administered orally for a long time (12 weeks). Fragment Ho1-205 protects against EAMG when given before disease induction and suppresses an ongoing disease when given at the acute and even during the chronic phase of EAMG in rats.

The effect of oral administration during the chronic phase of EAMG might be of special relevance to human $\mathrm{MG}$, which is a chronic disease. It is encouraging that appropriate AChR fragments could alleviate the symptoms of a chronic ongoing autoimmune process in rats that resembles the situation of patients with $M G$ at the time of their diagnosis. Therefore, oral administration of recombinant fragments corresponding to the domains of human AChR, encompassing major B- and T-cell epitopes, may have a potential of providing a safe and effective strategy for antigen-specific immunotherapy in myasthenia.

\section{Acknowledgments}

This research was supported by grants from The Association Française contre les Myopathies, The Muscular Dystrophy Association of America, and The Robert Koch-Minerva Center for Research in Autoimmune Diseases at The Weizmann Institute of Science. The authors thank A. Ben-Nun for fruitful discussions and C. Bar-Natan for excellent technical assistance.

1. Fuchs, S. 1979. Immunology of the acetylcholine receptor. Curr. Top. Microbiol. Immunol. 85:1-29.

2. Drachman, D.B. 1996. Immunotherapy in neuromuscular disorders: current and future strategies. Muscle Nerve. 19:1239-1251.

3. Drachman, D., Adams, R., McIntosh, K., and Pestronk, A. 1985. Treatment of experimental myasthenia gravis with cyclosporin A. Clin. Immunopathol. 34:174-188.

4. Balcer, L., McIntosh, K., Nicols, J., and Drachman, D. 1991. Suppression of immune responses to acetylcholine receptor by interleukin 2-fusion toxin: in vivo and in vitro studies. J. Neuroimmunol. 31:115-122.

5. McIntosh, K.R., Linsley, P.S., Bacha, P.A., and Drachman, D.B. 1998. Immunotherapy of experimental autoimmune myasthenia gravis: selective effects of CTLA4Ig and synergistic combination with an IL2-diphtheria toxin fusion protein. J. Neuroimmunol. 87:136-146.

6. Ahlberg, R., et al. 1994. Treatment of myasthenia gravis with anti-CD4 antibody: improvement correlates to decreased T-cell autoreactivity. Neurology. 44:1732-1737.

7. Newsom-Davis, J., et al. 1989. T-cell reactivity in myasthenia gravis. J. Autoimmun. 2(Suppl.):101-108.

8. Lisak, R.P., Levinson, A.I., Zweiman, B., and Kornstein, M.J. 1987. In vitro synthesis of IgG and antibodies to AChR by peripheral and thymic lymphocytes. Ann. NY Acad. Sci. 505:39-49.

9. Fuchs, S. 1999. Antigen-specific immunomodulation of experimental autoimmune myasthenia gravis. In The decade of autoimmunity. Y. Shoenfeld, editor. Elsevier Science Publishers. Amsterdam, the Netherlands. 255-260.

10. Bartfeld, D., and Fuchs, S. 1978. Specific immunosuppression of experimental autoimmune myasthenia gravis by denatured acetylcholine receptor. Proc. Natl. Acad. Sci. USA. 75:4006-4010.

11. Souroujon, M.C., Carmon, S., and Fuchs, S. 1992. Modulation of antiacetylcholine receptor antibody specificities and of experimental autoimmune myasthenia gravis by synthetic peptides. Immunol. Lett. 34:19-25.

12. Souroujon, M.C., Carmon, S., and Fuchs, S. 1993. Regulation of experimental autoimmune myasthenia gravis by synthetic peptides of the acetylcholine receptor. Ann. NY Acad. Sci. 681:332-334.

13. Wauben, M.H., et al. 1996. Inhibition of experimental autoimmune myasthenia gravis by major histocompatibility complex class II competitor peptides results not only in a suppressed but also in an altered immune response. Eur. J. Immunol. 26:2866-2875.

14. Balass, M., et al. 1993. Identification of a hexapeptide that mimics a conformation-dependent binding site of acetylcholine receptor by use of a phage-epitope library. Proc. Natl. Acad. Sci. USA. 90:10638-10642.

15. Yang, B., McIntosh, K.R., and Drachman, D.B. 1998. How subtle differences in MHC class II affect the severity of experimental myasthenia gravis. Clin. Immunol. Immunopathol. 86:45-58.

16. Souroujon, M.C., and Fuchs, S. 1986. Idiotypes and anti-idiotypes in experimental autoimmune myasthenia gravis. Ann. NY Acad. Sci. 475:81-93.

17. Killen, J., and Lindstrom, J. 1984. Specific killing of lymphocytes that cause experimental autoimmune myasthenia gravis by ricin-acetylcholine receptor conjugate. J. Immunol. 133:2549-2553.

18. Weiner, H. 1997. Oral tolerance: immune mechanisms and treatment of autoimmune diseases. Immunol. Today. 18:335-343.

19. Peterson, K.E., and Braley-Mullen, H. 1995. Suppression of murine experimental autoimmune thyroiditis by oral administration of porcine thyroglobulin. Cell Immunol. 166:123-130.

20. Gregerson, D.S., and Torseth,J.W. 1996. Oral tolerance and EAU: differences in mechanisms elicited by pre-versus post-priming feeding regimens. Invest. Ophthalmol. Visual Sci. 37:S1113. (Abstr.)

21. Bitar, D.M., and Whitacre, C.C. 1988. Suppression of experimental autoimmune encephalomyelitis by the oral administration of myelin basic protein. Cell Immunol. 112:364-370.

22. Higgins, P.J., and Weiner, H.L. 1988. Suppression of experimental autoimmune encephalomyelitis by oral administration of myelin basic protein and its fragments. J. Immunol. 140:440-445.

23. Nussenblatt, R.B., et al. 1990. Inhibition of S-antigen induced experimental autoimmune uveoretinitis by oral induction of tolerance with Santigen. J. Immunol. 144:1689-1695. 
24. Thompson, H., and Staines, N. 1990. Could specific oral tolerance be a therapy for autoimmune disease? Immunol. Today. 11:396-399.

25. Zhang, Z.J., Davidson, L., Eisenbarth, G., and Weiner, H.L. 1991. Suppression of diabetes in nonobese diabetic mice by oral administration of porcine insulin. Proc. Natl. Acad. Sci. USA. 88:10252-10256.

26. Guimaraes, V.C., et al. 1995. Suppression of development of experimental autoimmune thyroiditis by oral administration of thyroglobulin. Endocrinology. 136:3353-3359.

27. Wang, Z.Y., Qiao, J., and Link, H. 1993. Suppression of experimental autoimmune myasthenia gravis by oral administration of acetylcholine receptor. J. Neuroimmunol. 44:209-214.

28. Okumura, S., McIntosh, K., and Drachman, D.B. 1994. Oral administration of acetylcholine receptor: effects on experimental myasthenia gravis. Ann. Neurol. 36:704-713.

29. Ma, C.G., et al. 1995. Suppression of experimental autoimmune myasthenia gravis by nasal administration of acetylcholine receptor. J. Neuroimmunol. 58:51-60.

30. Karachunski, P.I., Ostlie, N.S., Okita, D.K., and Conti-Fine, B.M. 1997. Prevention of experimental myasthenia gravis by nasal administration of synthetic acetylcholine receptor T epitope sequences. J. Clin. Invest. 100:3027-3035.

31. Aharonov, A., Tarrab-Hazdai, R., Silman, I., and Fuchs, S. 1977. Immunochemical studies on acetylcholine receptor fraction from Torpedo californica. Immunochemistry. 14:129-137.

32. Lennon, V.A., Lambert, E.H., Leiby, K.R., Okarma, T.B., and Talib, S. 1991. Recombinant human acetylcholine receptor alpha-subunit induces chronic experimental autoimmune myasthenia gravis. $J$. Immunol. 146:2245-2248.

33. Barchan, D., Asher, O., Tzartos, S.J., Fuchs, S., and Souroujon, M.C. 1998. Modulation of the anti-acetylcholine receptor response and experimental autoimmune myasthenia gravis by recombinant fragments of the acetylcholine receptor. Eur. J. Immunol. 28:616-624.

34. Souroujon, M.C., Mochly-Rosen, D., Gordon, A.S., and Fuchs, S. 1983 Interaction of monoclonal antibodies to Torpedo acetylcholine receptor with the receptor of skeletal muscle. Muscle Nerve. 6:303-311.

35. Barchan, D., Souroujon, M.C., Im, S.H., Antozzi, C., and Fuchs, S. 1999. Antigen-specific modulation of experimental myasthenia gravis: nasal tolerization with recombinant fragments of the human acetylcholine receptor alpha-subunit. Proc. Natl. Acad. Sci. USA. 96:8086-8091.

36. Melamed, D., and Friedman, A. 1993. Direct evidence for anergy in T lymphocytes tolerized by oral administration of ovalbumin. Eur. J. Immunol. 23:935-942.

37. Mosmann, T. 1983. Rapid colorimetric assay for cellular growth and survival: application to proliferation and cytotoxicity assays. J. Immunol. Methods. 65:55-63.

38. al Sabbagh, A., Nelson, P.A., Akselband, Y., Sobel, R.A., and Weiner, H.L. 1996. Antigen-driven peripheral immune tolerance: suppression of experimental autoimmmune encephalomyelitis and collagen-induced arthritis by aerosol administration of myelin basic protein or type II collagen. Cell Immunol. 171:111-119.

39. Zipris, D., et al. 1996. Cytokine gene expression in islets and thyroids of $\mathrm{BB}$ rats. IFN-gamma and IL-12p40 mRNA increase with age in both diabetic and insulin-treated nondiabetic BB rats. J. Immunol. 156:1315-1321.

40. Tsuruta, H., et al. 1995. Quantitation of IL-1 beta mRNA by a combined method of RT-PCR and an ELISA based on ion-sensitive field effect transistor. J. Immunol. Methods. 180:259-264.

41. Shi, F.D., et al. 1998. Nasal tolerance in experimental autoimmune myasthenia gravis (EAMG): induction of protective tolerance in primed animals. Clin. Exp. Immunol. 111:506-512.

42. Drachman, D., Okumura, S., Adams, R., and McIntosh, K. 1996. Oral tolerance in myasthenia gravis. Ann. NY Acad. Sci. 778:258-272. 